\title{
Vaginal Vibrator as an alternative to Dilator in vaginal stenosis post pelvic radiotherapy in cervical cancer
}

\author{
Manjari Shah', Disha Tiwari ${ }^{2}$ \\ ${ }^{1}$ Attending Consultant, Department of Radiation Oncology, Jaypee Hospital, Noida, India, \\ ${ }^{2}$ Registrar, Department of Medical Oncology, Indraprastha Apollo Hospital, New Delhi, India
}

Sexuality, post cancer treatment is the most unattended aspect of patient doctor communication. Quality of life in a cancer survivor should be addressed without any inhibitions so that patient does not suffer from any kind of psychological distress. Vaginal stenosis is a well-known side effect of pelvic radiotherapy, which we have tried to address in our study. We have used vaginal vibrator as an alternative to vaginal dilator in two of our patients on experimental basis post vaginal dilator. Both the patients gave feedback that vibrator is comparatively easy to use and less painful. On follow up examination visits, their per vaginal examinations were easy to perform and visibly improved vaginal mucosal health. Psychosexual adjustment is an important domain for better quality of life. Vaginal vibrator is an unorthodox method which we have explored and found some promising results, in overcoming vaginal stenosis and adhesions post pelvic radiation. However, this aspect of treatment as well as method needs to explored.

Key words: Cervical Cancer; Pelvic Radiotherapy; Vaginal Stenosis; Sexual Dysfunction; Vaginal Dilator
Access this article online

\section{Website:}

http://nepjol.info/index.php/AJMS

DOI: $10.3126 /$ ajms.v12i10.37682

E-ISSN: 2091-0576

P-ISSN: 2467-9100

Copyright (c) 2021 Asian Journal of Medical Sciences

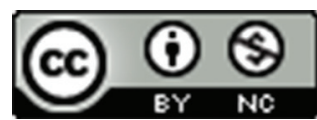

This work is licensed under a Creative Commons Attribution-NonCommercial 4.0 International License.

\section{INTRODUCTION}

Sexuality in India, for a long, has been considered as something to be discussed in hushed voices and behind closed doors. And if we talk about female sexuality, it has always been an elusive and contentious subject. For several decades now there's been an inherent enigma that surrounds female sexuality, manifested in a notion that has led to the understanding that women do not want or even need sexual gratification. Sexuality post-gynecological cancer treatment is important to address as 40\%-100\% of gynecological cancer patients will experience sexual complaints after treatment. ${ }^{1}$

The irony is that the two-common cancer in females in India are Breast and Cervical cancer are both sexually related organs and the taboo associated with sex and sexual organ remains the biggest problem. ${ }^{2}$ Statistics suggest that about 527,624 and 1,671,149 new cases of cervical and breast cancers are added every year. To this, India contributes about 122,844 cervical cancer and 144,937 breast cancer cases every year. ${ }^{2}$

Recent advancement in diagnosis, cancer treatment and technique has led to the increasing number of people surviving with cancer and as the survival increases our focus needs to shift to the morbidities associated with survival. ${ }^{3}$ The quality of life of a survivor has become of utmost importance in the current scenario and it also includes the sexual function in females who have won their battle with cancer. 
Most studies indicate that only about 15\% of gynecological cancer patients had been briefed on expected sexual dysfunction during and after treatment. ${ }^{4}$ Sexual dysfunction is a potential long-term complication of many cancer treatments including surgery, chemotherapy, and radiotherapy.

Radical treatment of cervical cancer in the form of both surgery as well as radiotherapy results in greater impairment of quality of life along with both physical and psychological effects. ${ }^{5,6}$ Radiotherapy is one of the main therapeutic modalities in cancer treatment, especially in gynecological cancer.

Sexual dysfunction due to radiotherapy have slightly worse sexual outcomes. ${ }^{7}$ An Indian study suggested sexual dysfunction from radiotherapy is mainly due to vaginal stenosis. ${ }^{8}$ Sexual dysfunction from surgery is mainly due to the shortened vagina, vaginal dryness, decreased libido. However, after radiotherapy, sexual dysfunction is caused by vaginal stenosis, leading to dyspareunia, difficulty in orgasm, and decreased sexual satisfaction. ${ }^{9}$ About a third of women suffer from vaginal stenosis after pelvic radiotherapy due to altered vaginal epithelium causing sexual dysfunction. ${ }^{10}$ Dilation might separate the adhesions formed by the denuded epithelium, thus possibly preventing stenosis. ${ }^{11}$ Three good surveys have shown an association between less stenosis and greater dilation frequency if dilation is commenced after the completion of the radiotherapy course. ${ }^{12-14}$

\section{MATERIALS AND METHODS}

Patients were prescribed an unconventional method i.e., vaginal vibrator in place of routinely used vaginal dilator (Figure 1) for correction of post radiotherapy vaginal stenosis in carcinoma cervix patients (Figure 2). Patients were advised to start using a vaginal dilator along with initiation of sexual activity two weeks after completion of radiotherapy. They were reviewed clinically on their first follow up which was three months later to completion of treatment.

Hereby presenting two cases suffering from sexual dysfunction post-treatment and the use of an unconventional treatment method in their management.

A 43 years old lady diagnosed with squamous cell carcinoma, FIGO Stage IIb, was taken up for concurrent chemoradiation for 5 weeks followed by 3 sessions of brachytherapy. When the patient came for the follow-up after 3 months, her pelvic examination was very difficult and painful. Whole-body PET-CT reported no evidence of disease. The patient was counseled regarding having frequent intercourse but she complained of having severe dyspareunia, the patient was given another option of vaginal dilation therapy along with lubricants but she was having difficulty in vaginal dilation.
The patient wanted to have normal sexual life and she was going through lots of emotional turmoil. She has negative impact of radiation treatment on her psychological, sexual and social life. At last, patient was advised a vaginal vibrator, which was an unconventional technique for the treatment of vaginismus and vaginal stenosis. The patient came on the next follow-up after 6 months she was able to resume her normal sexual life with the help of a vibrator. Patient was asked if she had any difficulty in using the vibrator and frequency of its use for documentation and future reference. As per the patient she could use the vibrator easily and as per the prescribed frequency. Her pelvic exam was normal and easy this time, no adhesions and stenosis were found.

Another case of 38 years old lady diagnosed with squamous cell carcinoma, FIGO Stage IIIa, was treated with concurrent chemoradiation for 5 weeks followed by 3 sessions of brachytherapy. The patient was explained in detail regarding the follow-up and need for a pelvic examination. She was counseled for frequent intercourse

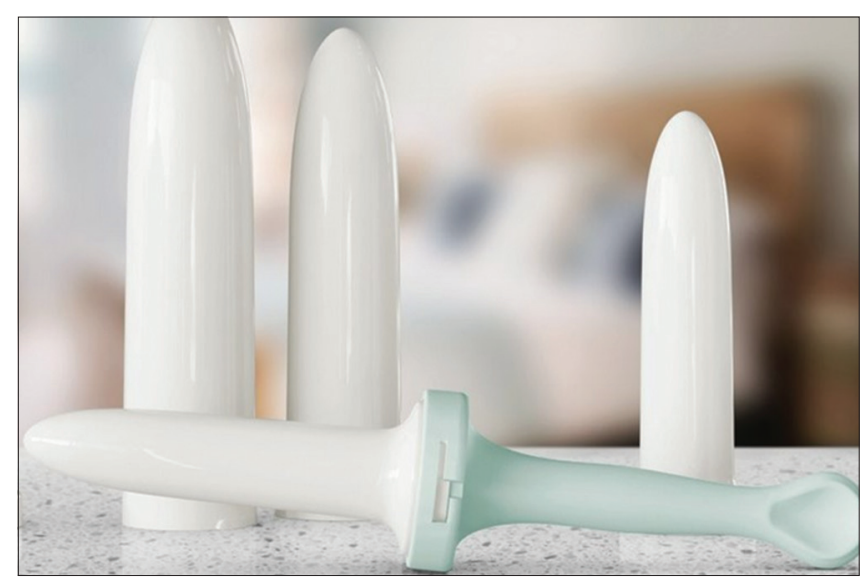

Figure 1: Vaginal dilator of different sizes made up of plastics (Pic Courtesy: Hope and Her)

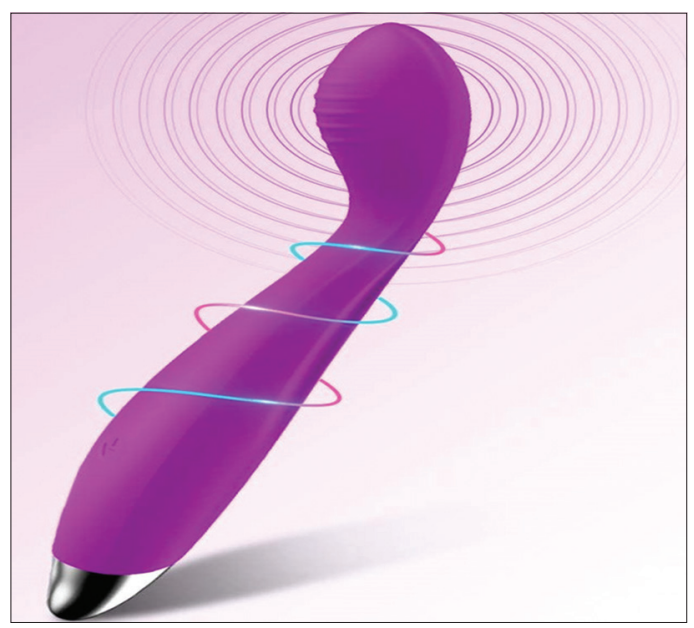

Figure 2: Vaginal Vibrator with different vibrating mode made up of silicone (Pic Courtesy: Amazon) 
after radiation to maintain the patency of the vagina. But patient was not sexually active post radiation treatment. She was explained regarding the use of a vaginal dilator. On follow-up, after 3 months patient comes with complaints that the dilator is very mechanical and painful. On her pelvic examination, there was adhesion present, and palpation of cervical was difficult, it was difficult to do pap smear test of the patient because of adhesions. The patient underwent CT Pelvis which showed no evidence of disease. This patient was also explained regarding the use of a vaginal vibrator, and on the next follow up when she came, she seems to be relaxed and happy with the use of a vibrator, according to her it was easy to manipulate in comparison to the dilator and it was pleasurable so the patients use it frequently. On six months follow up after radiation her pelvic exam was normal, per-speculum examination reveals no disease and pap smear sampling was easy for the patient this time.

Both the patients were advised to use a vaginal vibrator instead of vaginal dilator as both of them provided a history of difficulty in using a vaginal dilator. A vibrator advised for at least 3 to 4 times a week. Depth of use and intensity were the patient's-controlled parameters. Patients were also prescribed local anesthetic jellies in case of pain and discomfort. Counseling was done regarding the prohibition of sharing the device with another person to maintain strict hygiene or can be used with a barrier method. This device is suitable for women with any gynecological malignancy (carcinoma cervix, carcinoma endometrium) who has received radiation treatment either as post-operative or upfront. There is no absolute contraindication for its use and should be advised after properly educating the patient.

\section{DISCUSSION}

Vaginal vibrator is a newer mode of treatment which needs further research and evaluation. We have not found any study in which there is a direct comparison of vibrator and dilator was done in case of cervical stenosis post-radiotherapy, but one unpublished data by miles ${ }^{15}$ that have used vibrator instead of the dilator in case of vaginal stenosis. There was no difference in the outcome but this study had a high dropout rate and suffered from recruitment bias.

Benefits of Vaginal vibrator: There are many benefits of the vibrator that make it easier to use in comparison to the vaginal dilator. It is more flexible so can be manipulated easily, promotes healing of scar tissue by increasing blood supply to the areas, promotes natural vaginal lubrication, and is under user control in terms of depth, speed, and intensity. Gentle vibrations help the pelvic muscles to relax, like a massage, and stimulate the nerve ending in the clitoris, vulva, and vagina creating pleasure.
With the help of vibrator post pelvic radiation women can regain vaginal patency which also helps them in leading normal sexual life. Another advantage of using a vaginal vibrator and its successful outcome of maintaining patency is that it forbids unnecessary tests like CT-Scan as a followup assessment method and recommended clinical method for evaluation and PAP smear can be easily performed.

Guidelines for Vaginal Dilation: Routine vaginal dilation is advocated by $97 \%$ of UK radiotherapy centers. Surveys show that vaginal dilation with radiotherapy is standard British practice $^{16}$ and the UK guidelines recommended dilation "three times weekly for an indefinite period". ${ }^{17}$ Australian guidelines recommend dilation after brachytherapy "as soon as comfortably possible" and "certainly within four weeks" and "for three years or indefinitely, if possible" (Best Clinical Practice Guidelines 2009) ${ }^{18}$ USA practice guidelines by International Gynecological Cancer Society (IGCS). This recommended routine regular vaginal dilation after the inflammatory phase of radiotherapy had settled for two years, or at the clinician's discretion (Miles 2012). ${ }^{19}$

There are recommended guidelines from different countries for vaginal dilation but in India due to cultural and religious beliefs this is the last thing we want to talk about. There is a study by Shankar et al., ${ }^{8}$ who have reported that among women surviving after gynecological malignancies sexual dysfunction is morbidity unexplored in the Indian context because of cultural barriers. Despite the immense distress it causes in patients, sexual dysfunction is neither screened nor treated in the Indian scenario. Research has shown that up to $50 \%$ of women treated for cervix cancers have sexual dysfunction as they recover and become cancer survivors.

Symptoms related to sexual dysfunctions are often missed by oncologists during and post cancer treatment as patients in India are still not comfortable in talking about their sexual life.

Patient education, repeated counseling of both patient and her partner, pelvic muscle physiotherapy are some of the important aspects of sexual dysfunction management in post radiotherapy patients. According to a survey of 500 adults over the age of 25 , the most common reasons for not bringing up sexual issues were fear of the concern being dismissed (71\%), fear that the physician would be uncomfortable (68\%), and fear of a lack of treatment options $(76 \%) .{ }^{20}$

\section{CONCLUSION}

Cancer survivorship is a neglected domain in cancer management. Psychosexual adjustment after treatment completion is an imperative need. In this study, vaginal vibrator showed promising results in improving sexuality 
as well as maintain the patency of vagina for clinical assessment in patients who faces difficulties with vaginal dilators; however, this unorthodox treatment option needs further evaluation. Some consolidated data is required to include this option as part of management but we believe this study is a good start.

\section{REFERENCES}

1. Audette $\mathrm{C}$ and Waterman $\mathrm{J}$. The sexual health of women after gynecologic malignancy. J Midwifery Women's Health. 2010; 55(4):357-362.

https://doi.org/10.1016/j.jmwh.2009.10.016

2. Ferlay J, Soerjomataram I, Dikshit R, Eser S, Mathers C, Rebelo M, et al. Cancer incidence and mortality worldwide: Sources, methods and major patterns in GLOBOCAN. International Journal of Cancer. 2012; 136(5): E359-E386.

https://doi.org/10.1002/ijc.29210

3. Howlader N, Noone AM, Krapcho M, Neyman N, Aminou R, Altekruse SF, et al. SEER Cancer Statistics Review, 1975-2009 (Vintage 2009 Populations) Bethesda, MD: National Cancer Institute. 2012; Apr 30.

https://seer.cancer.gov/csr/1975_2017

4. Tsai T, Chen S, Tsai M, Su Y, Ho C and Su H. Prevalence and Associated Factors of Sexual Dysfunction in Cervical Cancer Patients. The Journal of Sexual Medicine. 2011; 8(6): 17891796.

https://doi.org/10.1111/j.1743-6109.2010.01745.x

5. Hendren S, O'Connor B, Liu M, Asano T, Cohen Z, Swallow C, et al. Prevalence of Male and Female Sexual Dysfunction Is High Following Surgery for Rectal Cancer. Annals of Surgery. 2005; 242(2): 212-223.

https://doi.org/10.1097/01.sla.0000171299.43954.ce

6. Juraskova I, Butow P, Robertson R, Sharpe L, McLeod C and Hacker N. Post-treatment sexual adjustment following cervical and endometrial cancer: a qualitative insight. Psycho-Oncology. 2003; 12(3): 267-279.

https://doi.org/10.1002/pon.639

7. White I, Faithfull S and Allan H. The re-construction of women's sexual lives after pelvic radiotherapy: A critique of social constructionist and biomedical perspectives on the study of female sexuality after cancer treatment. Social Science and Medicine. 2013; 76:188-196.

https://doi.org/10.1016/j.socscimed.2012.10.025

8. Shankar A, Prasad N, Roy S, Chakraborty A, Biswas A, Patil J, et al. Sexual Dysfunction in Females after Cancer Treatment: an Unresolved Issue. Asian Pacific journal of cancer prevention. 2017; 18(5): 1177-1182.

9. Thapa N, Maharjan M, Xiong Y, Jiang D, Nguyen T, Petrini M, et al. Impact of cervical cancer on quality of life of women in Hubei, China. Scientific Reports. 2018;8(1): https://doi.org/10.1038/s41598-018-30506-6

10. Denton A, Bond S, Matthews S, Bentzen S, Maher E and UK Link Gynaecology-Oncology Group, 2000. National Audit of the Management and Outcome of Carcinoma of the Cervix Treated with Radiotherapy in 1993. Clinical Oncology. 12(6): 347-353. https://doi.org/10.1053/clon.2000.9192

11. Hodgson D. Supportive Care in Radiotherapy Edited by Sara Faithfull and Mary Wells. Churchill Livingstone; 2003; ISBN 044306486 5; 400pp. Journal of Radiotherapy in Practice. 2003: 3(3): 163-164.

https://doi.org/10.1017/S1460396903000177

12. Bahng AY, Dagan A Brunner D and Lin LL. Determination of prognostic factors for vaginal mucosal toxicity associated with intravaginal high-dose rate brachytherapy in patients with endometrial cancer. International Journal of Radiation Oncology, Biology and Physics. 2012;82(2):667-673.

https://doi.org/10.1016/j.jijrobp.2010.10.071

13. Gondi V, Bentzen SM, Sklenar KL, Dunn EF, Petereit DG, Tannehill SP, et al. Severe late toxicities following concomitant chemoradiotherapy compared to radiotherapy alone in cervical cancer: an inter-era analysis. Journal of Radiation Oncology, Biology, Physics. 2012;84(4):973-982.

https://doi.org/10.1016/j.jrobp.2012.01.064

14. Law E, Kelvin J, Thom B, Riedel E, Tom A and Goodman K. Prospective study of vaginal dilator use adherence and efficacy following pelvic and intravaginal radiotherapy. Journal of Clinical Oncology. 2013; 31(15_suppl): 9524-9524. https://doi.org/10.1200/jco.2013.31.15_suppl.9524

15. Miles T. Evaluating vaginal dilator therapy after pelvic radiotherapy [PhD thesis]. Bristol, UK. The University of the West of England Bristol, Faculty of Health and Social Care. 2012.

16. White ID and Faithfull S. Vaginal dilation associated with pelvic radiotherapy: a UK survey of current practice. International Journal of Gynaecological Cancer. 2006; 16:1140-1146. https://doi.org/10.1136/ijgc-00009577-200605000-00029

17. Best practice guidelines on the use of vaginal dilators in women receiving pelvic radiotherapy. National Forum of Gynaecological Oncology Nurses Oxon: Owen Mumford, July 2005. https://doi.org/10.1186/1745-6215-14-39

18. GMCT Gynaecological Oncology Guidelines Review Group. Vaginal stenosis. Best Clinical Practice Gynaecological Cancer Guidelines Sydney: NSW Department of Health, 2009:16. [ISBN 978-1-74187-444-0]

19. Miles TP and Johnson N. Systematic literature review, a randomised trial and a preference trial challenging the practice of vaginal dilation during radiotherapy. $13^{\text {th }}$ Biennial Meeting of the International Gynaecological Cancer Society (IGCS 2010), Prague, Czech Republic. 2010.

https://doi.org/10.1002/14651858.CD007291.pub3

20. Marwick $C$. Survey says patients expect little physician help on sex. JAMA. 1999; 281: 2173- 2174.

https://doi.org/10.1001/jama.281.23.2173

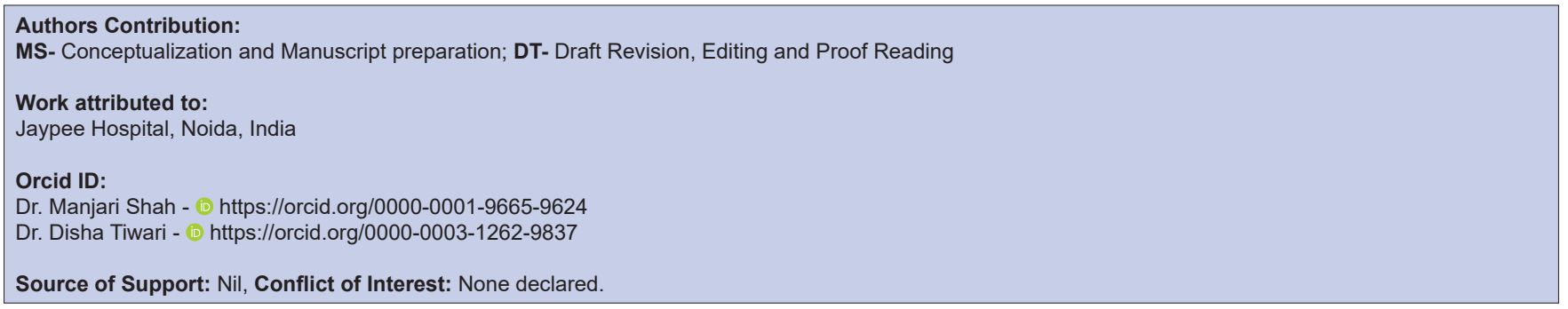

